\title{
Analysis of the ASDEX Upgrade 3-strap antenna with TOPICA code: Curved vs. flat 3D geometry
}

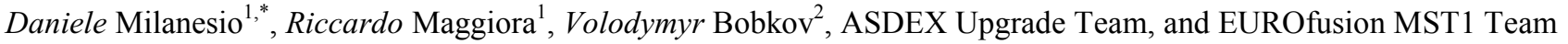 \\ ${ }^{1}$ Politecnico di Torino, Dipartimento di Elettronica e Telecomunicazioni (DET), Torino, Italy \\ ${ }^{2}$ Max-Planck-Institut für Plasmaphysik, Boltzmannstr. 2, 85748 Garching, Germany
}

\begin{abstract}
Before the 2015/2016 experimental campaign, the ASDEX Upgrade (AUG) 2-strap ICRF antennas with tungsten-coated limiters were replaced by 3-strap antennas. The main goal of the 3-strap launcher was to reduce the release of tungsten (W) in order to improve the ICRF operation, which appeared to be troublesome after the all-W wall installation [1]. In this paper, we analyse the behaviour of the 3-strap antenna with the help of TOPICA code [2], a numerical tool able to take into account a realistic antenna geometry and an accurate plasma description. By loading an experimental plasma profile from the AUG campaign, we characterize the antenna both in terms of input parameters and of radiated fields. In particular, we compare TOPICA results obtained with a simplified 3D flat model adopted during the design phase with the exact 3D curved geometry installed on the AUG experiment. In particular, the curved model predicts a lower coupling to plasma and higher RF electric fields with slightly different distribution in front of the launcher. The capability to include a fully $3 \mathrm{D}$ curved model is of great importance to correctly account for all geometrical effects on the antenna performances. The advantages and disadvantages of both geometrical representations are eventually outlined, trying to estimate how the curvature of the antenna can affect code predictions. Comparisons between measured experimental results and simulated ones are presented in [8].
\end{abstract}

\section{Introduction and objectives}

TOPICA [2] is a numerical tool realized for the 3D/1D simulation of Ion Cyclotron (IC) antennas, i.e. accounting for realistic 3D geometry with an accurate 1D plasma model. While referring the interested reader to [2] for a more detailed analysis of TOPICA formulation, we would like to enlighten here only the features that have been exploited in this paper. In particular, the code permits to compute both the input parameters of an IC launcher, and hence the power coupled to plasma, and the electric field distribution everywhere inside the antenna enclosure and in the plasma column. Moreover, with the help of postprocessing tools it is possible to determine the local electric field value thought to be the most important element for driving Radio Frequency (RF) sheaths, and to directly verify the impact of the geometrical accuracy of the antenna on the field values themselves. From the plasma side, 1D FELICE code [3] has been adopted, affording density and temperature profiles, and finite Larmor radius effects.

Provided the mentioned tool, the goal of this paper is to compare TOPICA results obtained with a simplified 3D flat model of the new 3-strap AUG antenna (adopted during the design phase) with the real 3D curved geometry installed on the experiment. Both the power transferred to plasma and the local electric fields will be analyzed in order to show the differences between the two simulated versions of the same launcher. A set of nine artificial plasma profiles will be adopted for this task, together with an experimentally measured one from 2015 campaign.

\section{Antenna models and plasma profiles}

As already mentioned, before the 2015/2016 experimental campaign, the AUG 2-strap ICRF antennas with tungsten-coated limiters were replaced by 3 -strap antennas, whose main goal was to reduce the release of tungsten in order to improve the ICRF operation [1]. Most of the 3-strap antenna design was actually performed with a simplified 3D flat model with the help of HFSS suite [4] and TOPICA; Figure 1 reports the TOPICA version of the 3D flat launcher.

The real 3D curved model has been tailored for an electromagnetic simulation code only quite recently; it is worth mentioning that this model was directly imported from the technical drawings and, therefore, all the geometrical details of the antenna are consistent with the launcher actually installed on the machine. Figure 2 reports the $3 \mathrm{D}$ curved version of the launcher simulated with TOPICA.

For sake of comparison, Figure 3 also reports the real antenna model. 


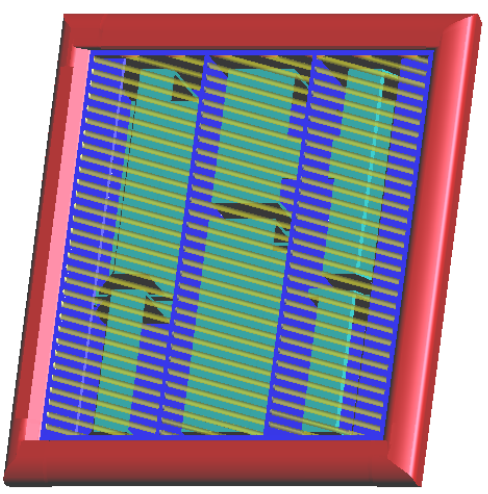

Fig. 1. 3D flat model of the 3-strap AUG antenna.

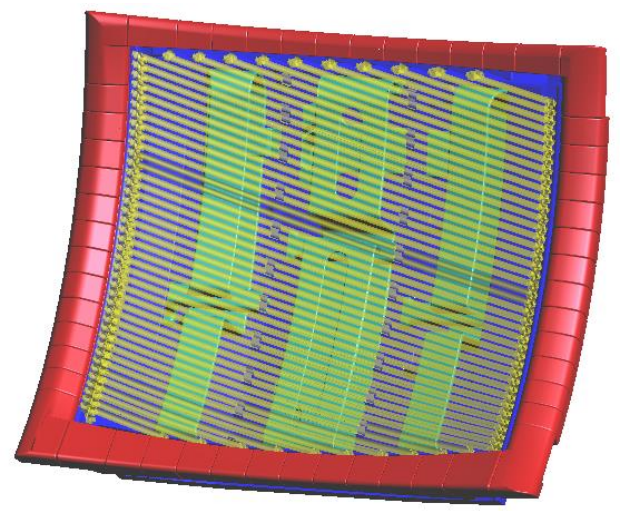

Fig. 2. 3D curved model of the 3-strap AUG antenna.

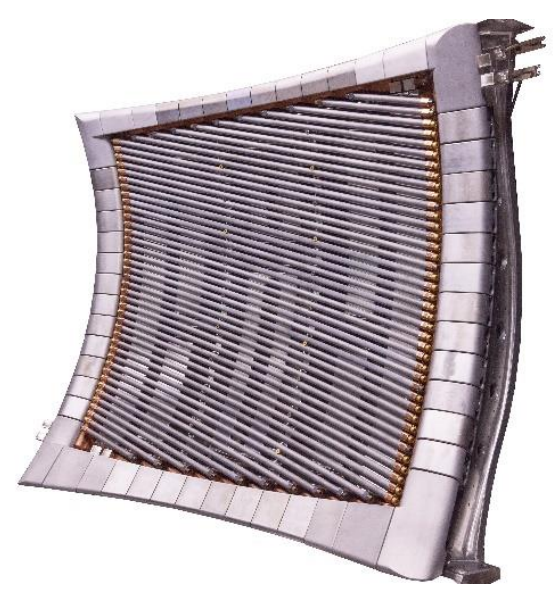

Fig. 3. Real antenna model installed on AUG

The reader may immediately notice that not only the introduced curvature represents quite a difference between the two models, but also several other geometrical details appear as simplified in the flat antenna geometry, in particular in the plasma exposed antenna region. Despite this, we tried to reduce any source of difference due to TOPICA between the two groups of simulations: we imposed a similar mesh density on both antennas (about $4 \mathrm{~cm}$ resolution), we loaded the same density profiles at the same distance from the radiating elements (about $4.7 \mathrm{~cm}$ ), i.e. keeping constant the strap-cutoff distance in both cases, we computed the electric field distribution on the same surface (located about $3 \mathrm{~mm}$ in front of the limiters). The different geometrical accuracy is clearly witnessed by the mesh requirements: the flat antenna model is discretized by approximately $65 \mathrm{k}$ unknowns while the curved one requires more than $250 \mathrm{k}$ unknowns to account for all geometrical details. This translates in heavier computational requirements too, namely from 576 CPUs to 900 CPUs for about 5 hours, on the new Marconi High Performance Computing (HPC) system at CINECA [5].

Nine "artificial" density profiles were loaded for this comparison, as documented in Figure 4. These profiles sets correspond approximately to the cases for various gas injection sources described in [6]. The same temperature profile was loaded for both ions and electrons in all cases. In addition to them, a measured profile, namely shot 31515 , was also included into the simulation database.

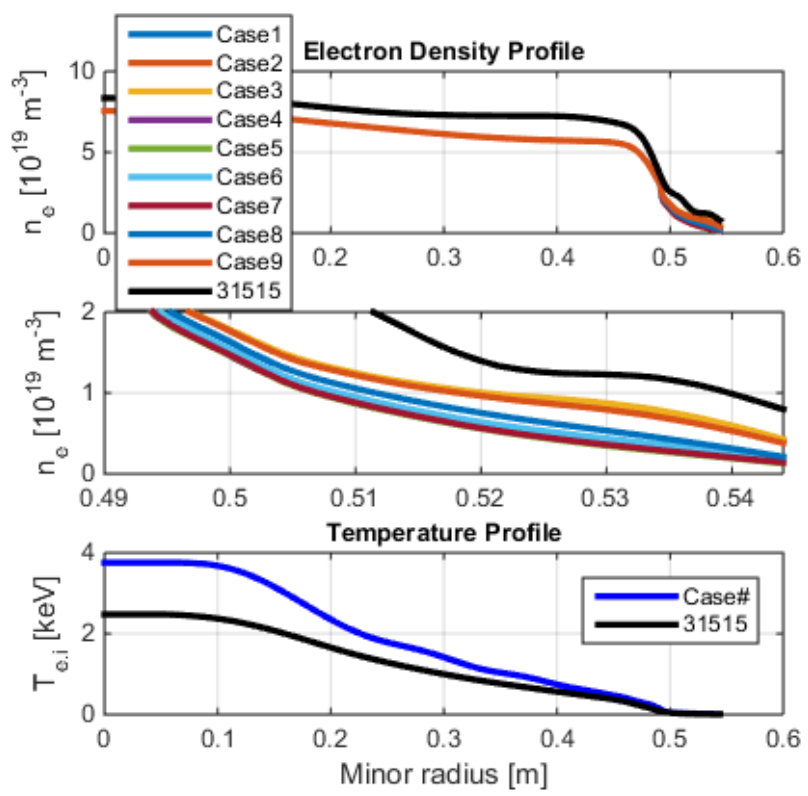

Fig. 4. Loaded plasma profiles: electron density (top) with zoomed view of the edge density profile (middle) and ion/electron temperature profile (bottom).

\section{Analysis of the results}

Provided the aforementioned loadings, the two geometries are first compared in Figure 5 in terms of power transferred to plasma, assuming infinite coaxial lines connected to the two antenna ports withstanding a maximum voltage of $30 \mathrm{kV}$ and imposing $0 \pi 0$ phasing at the straps. The working frequency is $30 \mathrm{MHz}$. Figure 5 reports the same relative behavior for both geometries but the difference in loading is approximately of a factor of 2. For both geometries the measured plasma profile, i.e. shot number 31515, generates a higher coupled power which is basically due to the slightly higher edge density with respect to the other "artificial" cases.

The reader should remind that, while TOPICA can handle a fully curved geometry, the coupled plasma code, namely FELICE, is a 1D model. The two codes are joined together with the help of a procedure named 
stretching in [7, App. A], which allows to flatten the curved interface between the antenna and the plasma. Thanks to this procedure, the simulated results obtained with the curved antenna geometry better match the experimental evidence. A further improvement could be to directly couple a 3D plasma model.

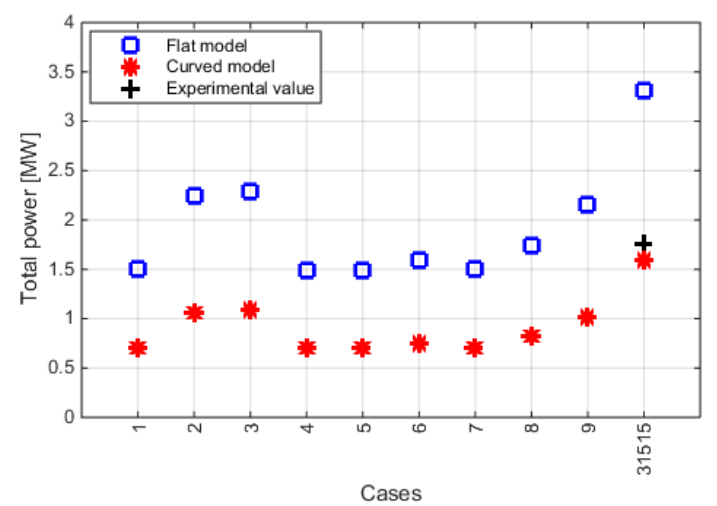

Fig. 5. Power transferred to plasma for different plasma cases with flat (blue squares) and curved (red stars) geometries. The black cross reports the experimental measure.

To conclude the comparison between the flat and curved launchers, the parallel electric field is also calculated. $0 \pi 0$ phasing is imposed at the straps with $1 \mathrm{MW}$ of total power transferred to plasma; besides, to minimize the parallel electric field in front of the limiters, a ratio of two is imposed between the power radiated by the central strap and the one radiated by the sum of the two outer ones. Figure 6 and Figure 7 reports the real part of the electric field parallel to the magnetic field lines $\left(11^{\circ}\right.$ tilted respect to the toroidal axis, shown on the picture with a dashed line) for the flat and curved models respectively, loading Case 3 plasma profile.

The overall pattern of the parallel electric field distribution is not that different for both geometries, even though one should notice that the curved model shows a more pronounced field distribution on the central part of the antenna.

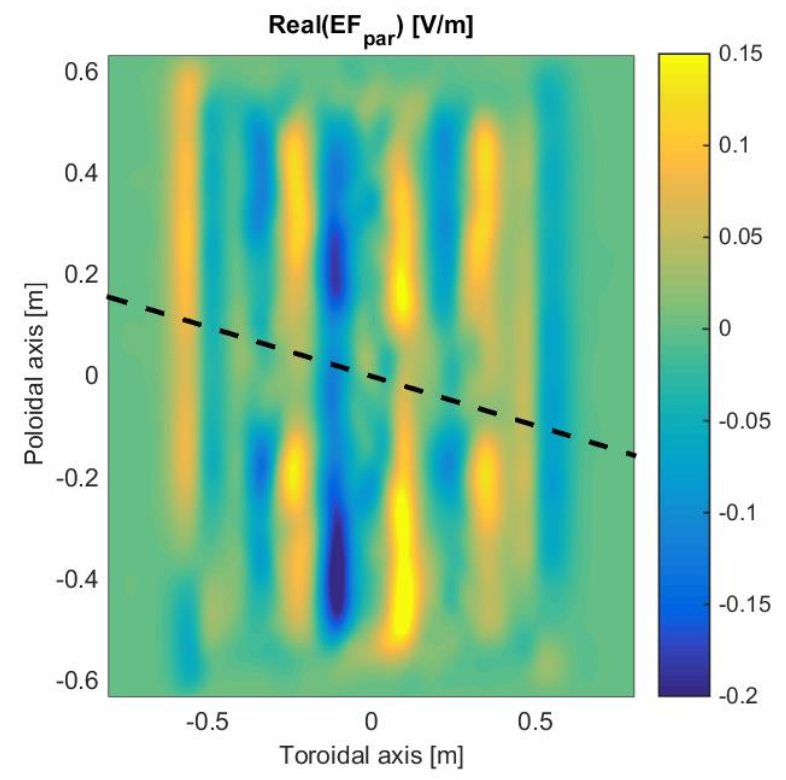

Fig. 6. Parallel electric field computed with $0 \pi 0$ input phasing for the flat antenna model, assuming $1 \mathrm{MW}$ of delivered power.

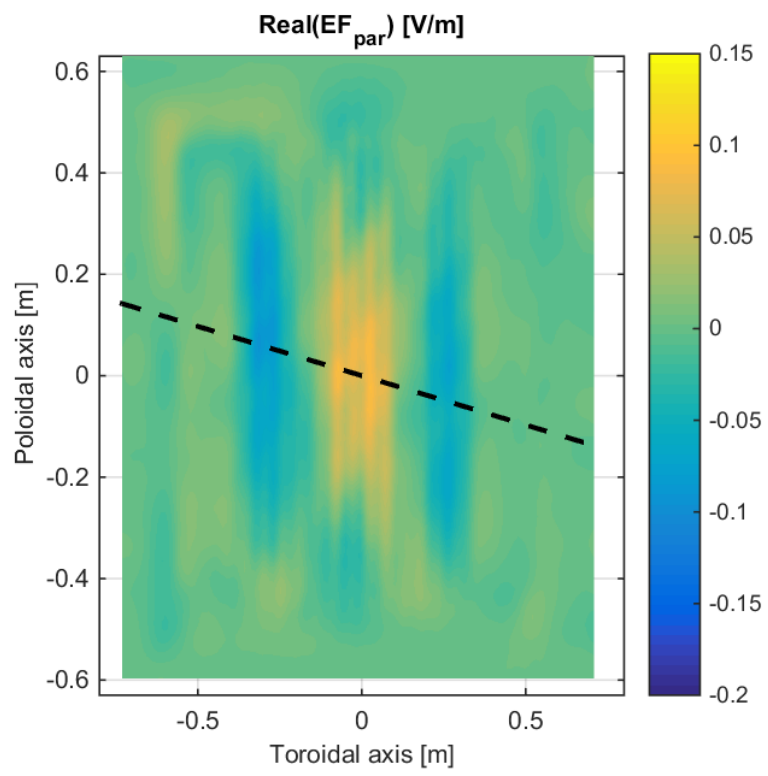

Fig. 7. Parallel electric field computed with $0 \pi 0$ input phasing for the curved antenna model, assuming $1 \mathrm{MW}$ of delivered power.

As fully detailed in [9], recent experiments proved that the quantities relevant for antenna-plasma interactions cannot be described by a single value for a given magnetic field line. Therefore, instead of computing the integral of the parallel electric field along the magnetic field lines crossing the antenna, we preferred to follow the approach outlined in [9], namely to determine the average electric field in front of the limiters, i.e. the most protruding conducting structures. The parallel component of the electric field is still taken into account, but for each poloidal coordinate in front of both limiters, its value is averaged along the toroidal extension of the limiter itself. Figure 8 and Figure 9 show the so obtained curves for the antenna left and right limiters, respectively, loading three plasma profiles of the database.

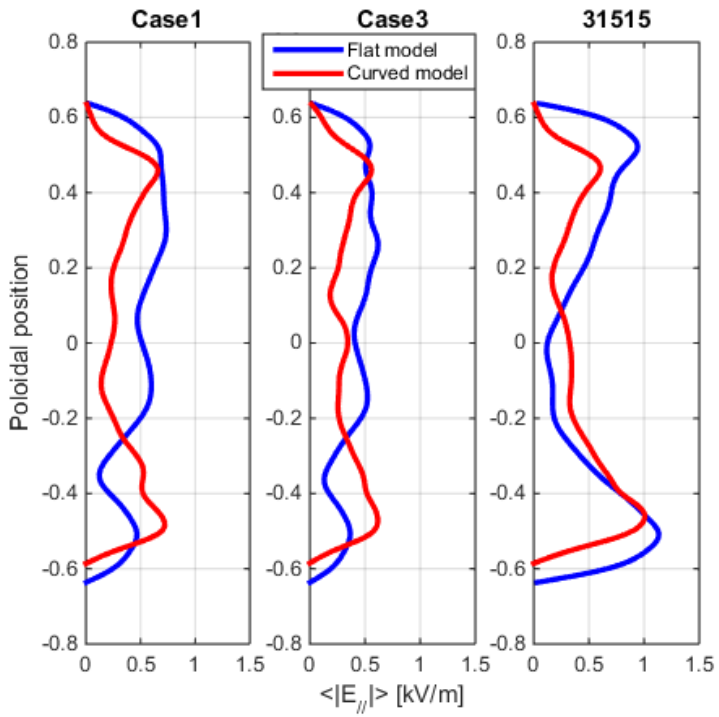


Fig. 8. Averaged electric field (parallel component) computed in front of the left limiter (seen from plasma) with $0 \pi 0$ input phasing for both geometries, assuming $1 \mathrm{MW}$ of delivered power.

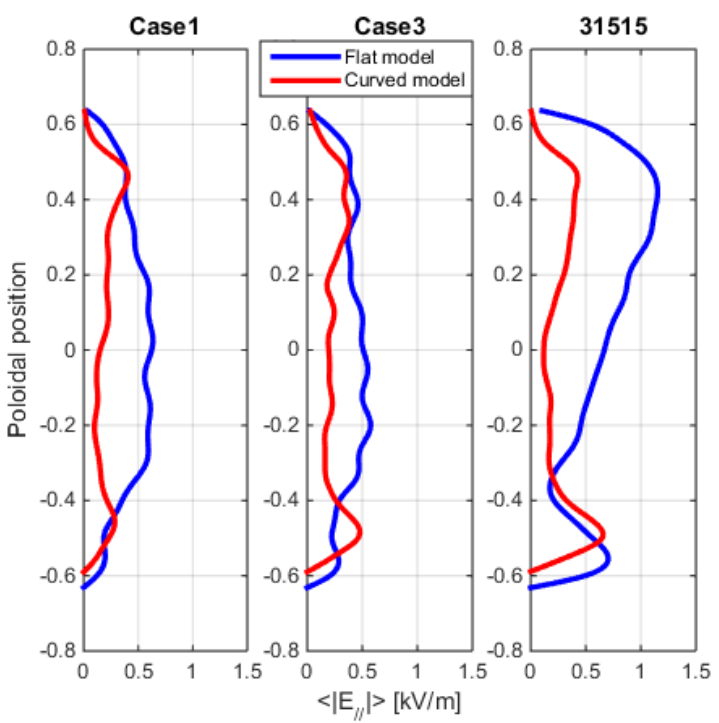

Fig. 9. Averaged electric field (parallel component) computed in front of the right limiter (seen from plasma) with $0 \pi 0$ input phasing for both geometries, assuming 1MW of delivered power.

Both the value and the position of the top and bottom peaks and, more generally, the poloidal behavior are rather different for the two geometries. At least for the maxima poloidal position, one should take into account that the flat model can be seen as the results of a stretching of the curved one; as a consequence, it is not surprising to observe a shift of the maxima towards the poloidal extremities of the plot. The reader should notice that the averaged parallel field shares the same qualitative pattern on both limiters in case of the curved geometry, while a strong asymmetry can be observed when looking at the flat launcher. This may be partially explained by the fact that, even though the total coupled power is identical, the ratio between the central and the outer straps is slightly different for the two geometries. Eventually, one should also observe that, for the setup adopted for this last analysis (the electric fields are computed few millimeters from the limiter front surface), the behavior of the electric fields reported in Figure 8 and Figure 9 follows (and it is indeed determined) the electric current distribution flowing on the side limiters.

We refer the interested reader to [8] for the analysis of the antenna behavior during the experiment and the comparison with numerical predictions.

\section{Conclusions}

The detailed analysis carried out with the help of TOPICA code indicates that a flat model can be a reasonably good approximation of a real curved launcher even though some differences still remain. It is unfortunately impossible for the time being to identify general rules to quantify how the adoption of a curved model instead of a flat one influences the simulated results except for a general reduction of the loading and a significant modification in the poloidal behavior of the RF sheaths. It is also clear that the differences between the simulated results obtained with a curved model instead of a flat one strongly depends on the specific antenna geometry and must be evaluated case by case.

This said, we firmly believe that a realistic curved antenna geometry should always be analyzed during any design process, especially if one is interested in more localized phenomena, such as RF sheaths, where even the smallest details can have an influence on the final result.

This work has been carried out within the framework of the EUROfusion Consortium and has received funding from the Euratom research and training programme 2014-2018 under grant agreement No 633053. The views and opinions expressed herein do not necessarily reflect those of the European Commission. The authors gratefully acknowledge the contribution of $\mathrm{W}$. Zhang concerning the profile generation. The complete list of all MST contributors can be find in "Overview of progress in European Medium Sized Tokamaks towards an integrated plasma-edge/wall solution" by H. Meyer et al., to be published in Nuclear Fusion Special issue: Overview and Summary Reports from the 26th Fusion Energy Conference (Kyoto, Japan, 17-22 October 2016).

\section{References}

1. V. Bobkov et al., Nucl. Fus. 56, 084001 (2016)

2. D. Milanesio et al., Nucl. Fus. 49, 115019 (2009)

3. M. Brambilla, Plasma Phys. Control. Fusion 35, 4162 (1993)

4. V. Bobkov et al., Nucl. Fus. 53, 093018 (2013)

5. www.hoc.cineca.it/hardware/marconi

6. W. Zhang, Nucl. Fusion 56, 036007 (2016)

7. V. Lancellotti et al., Nucl. Fus. 46, S476-S499 (2006)

8. V. Bobkov et al., Characterization of 3-strap antennas in ASDEX Upgrade, this conference

9. V. Bobkov et al., Plasma Phys. Control. Fusion 59, 014022 (2017) 